\title{
Efficient and Scalable Ant Colony Optimization based WSN Routing Protocol for IoT
}

\author{
Afsah Sharmin*, Farhat Anwar, S M A Motakabber \\ Faculty of Engineering, Department of Electrical and Computer Engineering, International Islamic University Malaysia, Kuala \\ Lumpur, 53100, Malaysia
}

\begin{tabular}{l} 
A R T I C L E I N F O \\
\hline Article history: \\
Received: 31 August, 2020 \\
Accepted: 21 December, 2020 \\
Online: 28 December, 2020
\end{tabular}

Keywords:

Wireless Sensor Networks

IoT

Routing algorithms

$A C O$

Energy consumption

\begin{abstract}
A B S T R A C T
IoT integrates and connects intelligent devices or objects with varied architectures and resources. The number of IoT devices is growing exponentially. Due to the massive wave of IoT objects, their diversity and heterogeneity among their architectures, the existing communication protocols for wireless networks become ineffective in the context of IoT. Wireless Sensor Network (WSN) has the potential to be integrated to the internet of things (IoT). The issues of the routing of WSNs impose nearly similar prerequisites for IoT routing technique. Most of the traditional routing protocols are not appropriate for WSNs and IoT because of resource constraints, computational overhead and environmental interference and do not take into account the different factors affecting energy parameter and do not accommodate node mobility. Routing algorithms must ensure the data transmission in an efficient way, having proper knowledge of the IoT system. For this reason, many intelligent systems have been utilized to design routing algorithms to handle the network's dynamic state. In this paper, an ant colony optimization (ACO) based WSN routing algorithm for IoT has been proposed and analyzed to enhance scalability, to accommodate node mobility and to minimize initialization delay for time critical applications in the context of IoT to find the optimal path of data transmission, improvising efficient IoT communications. The proposed routing algorithm is simulated using MATLAB for performance evaluations. The evaluation results have recorded an improvement in conservation of energy, of almost $50 \%$ less consumed energy even with an increase in the number of nodes, by comparing with an existing routing technique based on ant system, a current routing protocol for IoT and the conventional ACO algorithm.
\end{abstract}

\section{Introduction}

With the widespread use of IoT devices, the issue of designing efficient routing protocol has attracted more attention in networking research. This paper is an extension of work originally presented in ICOM'19 [1].

According to the prediction of $\mathrm{Cisco}^{\mathrm{TM}}$ IBSG [2], by 2020, 50 billion devices will be connected to the Internet. IoT provides network connectivity between these smart devices everywhere and all times. The emergence of a new ubiquitous computing era has been created due to the evolution of wireless networks and sensor technologies, allied to the increasing demand for new IoT applications for the provision of smart services [3]. In this context, WSNs play an essential role to the expansion of IoT while

${ }^{*}$ Corresponding Author: Afsah Sharmin, Faculty of Engineering, Department of Electrical and Computer Engineering, International Islamic University Malaysia, 53100 Kuala Lumpur, Malaysia, Tel: +60167101538, afsahsharmin@gmail.com providing ubiquity of networks with smart and low-cost devices that are easy to deploy. In an IoT system, a large collection of autonomous and dynamic sensor nodes are used to gather information by detecting physical parameters, communicate and cooperate with their environment and send their data to the internet. Sensor nodes have the ability of self-organization and sensor networks function in a distributed way [4]. IoT with the integration of WSNs, have a wide range of applications spaces that shape human life and also have impact on economic benefits. The physical domain of IoT is presented through the connected networks of objects and nodes utilizing wireless sensors. A large number of small devices surround the environment help to manage the physical world by sensing, processing, communicating and analyzing the data in the IoT network system [5]. The development of new applications and technologies are drawing attention from the research perspective, such as smart home, environmental monitoring, healthcare, transport, agriculture, offices, buildings and smart cities etc. 
Designing efficient routing protocol is the key factor to improve the energy efficiency, data transmissions, scalability and prolong network lifetime in WSNs and IoT. However, several considerations are required for resource-constrained network system, such as energy efficiency, scalability, security, autonomy, computational complexity, environmental constraints for wireless link, node mobility, the QoS (quality of service) requirement for a particular application, during IoT routing. The sensed information ought to be sent to the base station for further operations in various IoT applications through the competent forwarding mechanism while keeping in consideration different functionalities of IoT objects states.

Until now, there is a large number of routing algorithms have been proposed by the research community, considering the energy parameter, yet its exploitation is not well thought-out [6]. The existing node energy and the distinctive components affecting this energy parameter need to be considered to spare node energy and enhance the communication quality of the network. The node's lifetime relies upon the battery-life to a huge range, and the irrational energy utilization will effectuate the system to expire early and decrease the lifetime of the network [7]. Hence the key research issue is the designing of efficient route calculation algorithm that can ensure efficient information communication within IoT while maintaining scalability. Most of the traditional routing protocols are not adaptable other than energy efficient, if the varied difficulties in various applications are considered or due to the dense and complex conditions and a wide range of radio obstruction. Many intelligent systems including the working mechanism of the biological systems have been employed for designing routing algorithms to handle the network's dynamic state while keeping pace with energy efficiency during information communication [8]. Ant colony optimization (ACO) based algorithms emphasize the design of routing protocols that are robust, adaptable and scalable [9]. The coordination of ants depends on the ability of self-organization that ant colony optimization based swarm intelligence techniques possess [10]. The probabilistic approach of ACO is used to determine the routes, and the pheromone update formulation is used for further updating of the pheromone trail [11].

An ant colony based routing technique, named EICAntS, for effective communications within IoT is presented in [12]. This algorithm considers the energetic parameters. The pheromone estimations in the ant system is related here by the calculated global efficacy factor. An improvement with regard to network lifetime and conservation of energy is shown from the evaluation results. The algorithm does not include the heuristic information and pheromone update strategy consisting of pheromone evaporation rate and/or the amount of pheromone deposited. The energy effect precisely addresses the data class handled by the node and does not specify the different factors affecting energy parameter such as the energy consumption in free-space and multipath fading standard of wireless communication as well as no particulars are provided on how to compute the nodes' energy level. The REL routing technique for IoT applications, which is focused on energy and link quality information, is proposed in [13]. Testbed experiments are used besides simulation for the evaluation where REL increases the system and network lifetime, reliability, energy-efficiency, QoS of IoT applications and reduces packet loss rate. In addition, it offers a path determination system which is basically an end to end route. For this purpose, it depends on cross-layer data with insignificant overhead. Furthermore, it permits information transmission with a reasonable appropriation of wireless and remote assets. A piggyback and on-demand system guides nodes to become energy proficient where the residual energy is sent to their neighboring nodes. Nonetheless, no enhancement system is utilized in contradiction of ant based protocol, which utilizes the ant colony system.

In [14], LEACH-MA protocol is proposed which is based on modified ACO. The residual energy parameter is used along with LEACH (Low-energy adaptive cluster hierarchy routing) protocol for the selection of current cluster head. The measure of energy consumption is minimized by this algorithm. The energy and distances are combined in this approach for choosing the cluster head. But no facts have been provided on the threshold value for selecting the cluster head. In [15], a hybrid tree-based search approach, called ANT-BFS, is proposed to discover the optimum route for information communication. This scheme integrates breadth first search with ACO to minimize the amount of energy consumption. However, the memory and computational time requirements may arise some issues for this technique in case the cluster head and the sink are located far from each other, so it might not be suitable for large scale. Content based routing (CCR) protocol is presented in [16] that ensures reliability of information transmission for IoT applications. It utilizes the process of data aggregation and ensures good load balancing. This method transmits data based on the message content and incorporates link quality information. The traffic reduction gain is achieved by this technique, where an objective function is used by the nodes for routing of the heterogeneous sorts of content. Each node builds a distinct routing entry by using the content to select the next node for transferring the data via the certain reliable communication link. The energy consumption has been conserved by this method by forwarding aggregated data to selective nodes. However, no details were provided on the estimation of the reliability while taking into consideration of the parameters that are also unspecified for this reliability.

In [17], an ant-based routing algorithm considering the energy supervision is presented. Reward and punishment technique are adopted with the pheromone update rules. The energetic parameter is considered and this technique increases network lifetime and energy-efficiency. However, various factors of energy utilization are required to be addressed. The node delay parameter is used here, but no details were found on the delay factor. An improvised energy saving ant colony based routing protocol for sensor networks is proposed in [18]. Three phases are introduced in this multipath protocol, such as discovery of neighbor through link information, transmission of packets through EWMA (exponentially weighted moving average) technique and efficient $\&$ reliable end to end delivery. The simulation results indicate that the proposed routing protocol for dynamic networks stands efficient in general performance, particularly regarding energy efficiency and throughput when contrasted with standard and other novel proposed routing algorithms. A multi-constrained technique that ensures QoS, known as IAMQER focused on ant colony, is proposed in [19]. The simulation results show that average energy consumption is minimized and packet delivery ratio is increased by utilizing this technique. Also, a route evaluating function is presented here. But IAMQER strategy might suffer longer processing delay as it is based on traditional ACO method. 
This paper is an extension of work initially reported in [20], where the necessary parameters for communication process have been taken into account by the proposed method, such as mobility and energy parameters. Most of the proposed routing algorithms have addressed merely some communication parameters that are not adequate enough to enhance the communication quality needed by the energy constrained IoT applications. The important communication parameters must be considered by the mechanisms that mediate in the correspondence procedure, for instance routing, for resolving the issues of routing and for efficient communications in the IoT network system. The proposed protocol has utilized the advantages of the ant system to discover the optimum path for information communication and to improve communication quality within an IoT system.

\section{System Model}

\subsection{The Network Model}

The considered network model consists of $M$ sensor nodes with random distribution in an $L \times L$ rectangular area. The proposed algorithm comprises the system model for IoT communications which is designed with several nodes or sensors $M_{i}$. The adopted graph $\mathrm{G}$ with the nodes and connecting links has constructed the network. It is assumed that the sensor nodes have the same computation and preliminary energy. The nodes can update the information about its neighbors. The received signal strength indication (RSSI) is used to compute the approximate distance of the senders by the nodes. For that calculation, the transmitted power of the objective is acknowledged.

\subsection{Proposed ACO Algorithm}

The most pheromone path is chosen as the shortest and optimal path in the traditional ACO algorithm [11] and the energy parameter is not considered. Hence the network's node energy on that path reduces abruptly and lessens the entire network's lifespan. Furthermore, novel routing protocols are required to manage the overhead of mobility. As the topological changes for the mobility of the sensor nodes and the sink nodes generate frequent updates in the network, which may drain the node energy extremely in an energy-constrained system. This paper expands our research works presented in [1] in terms of network performance to improve communications within IoT. The proposed system of ours [21] has been investigated more here to enhance scalability, to accommodate node mobility, and to minimize initialization and processing delay for time critical applications in the context of IoT. An improved network routing algorithm based on ACO is proposed here by analyzing the nodes' balanced consumption of energy.

At the place of node $i$ and time $t$, each ant $m$ will adhere to the following probabilistic formula to select the next node $j$ being the forwarding node of the subsequent route for the enhancement that is proposed of the ACO algorithm of ours [20] for the next hop routing:

$$
\begin{aligned}
& P_{i j}^{m}= \\
& \left\{\begin{array}{c}
\frac{\left[\tau_{i j}(t)\right]^{\alpha}\left[\eta_{i j}(t)\right]^{\beta}\left[\vartheta_{i j}(t)\right]^{\gamma} E_{j}}{\sum_{\text {scallowed }}\left[\tau_{i s}(t)\right]^{\alpha}\left[\eta_{i s}(t)\right]^{\beta}\left[\vartheta_{i s}(t)\right]^{\gamma} E_{s}} \quad, j \subset \text { allowed }_{m} \\
0, \text { others }
\end{array}\right.
\end{aligned}
$$

here,

$$
\eta_{i j}(t)=\frac{1}{d_{i j}}
$$

where $\tau_{i j}(t)$ and $\eta_{i j}(t)$ are the amount of pheromone and heuristic information on edge $(i, j)$ respectively and $\eta_{i j}(t)$ is typically $1 / d_{i j} . \alpha$ and $\beta$ are two parameters that control the influence of the pheromone intensity and heuristic information respectively. $d_{i j}$ is the distance between $i$ and $j$. The average mobility parameter is used to calculate the stability factor, $\vartheta_{i j}(t)$, where $\gamma$ is the mobility constant. $E_{j}$ is the node residual energy that ant $m$ will visit.

To avoid faster local convergence in case large amount of pheromone deposition happens on the routes, the pheromone update is required to improve as well. So, the amount of pheromone is updated and limited by incorporating a threshold value and can be obtained as follows:

$$
\begin{gathered}
\tau_{i j}(t+1)=\left\{\begin{array}{c}
T, \quad \tau_{i j}(t+1)>T \\
(1-\rho) \tau_{i j}(t)+\Delta \tau_{i j}(t)
\end{array} \quad\right. \text { else } \\
\Delta \tau_{i j}(t)=\sum_{k=1}^{m} \Delta \tau_{i j}^{k}
\end{gathered}
$$

where $\rho$ is the pheromone coefficient for evaporation, $\rho \in(0,1), T$ represents the threshold value used to limit excessive pheromone deposition, $\Delta \tau_{i j}(t)$ is the increased pheromone concentration of edge $(i, j)$, usually given by,

$$
\Delta \tau_{i j}^{k}=\left\{\begin{array}{rr}
\frac{R}{L_{k}} & \text { if } k \text { th ant uses the edge }(i, j) \\
0 & \text { otherwise }
\end{array}\right.
$$

where $R$ represents strength of pheromone, $L_{k}$ denotes the path length of the $k^{\text {th }}$ ant.

\subsection{Energy Model}

The energy model of wireless communications that is presented in [22] is incorporated by the proposed system. The freespace and multi-path fading model are utilized here depending upon the distance $d$ in between the sending and receiving nodes and a threshold value, $d_{0}$. The following equations for energy consumption for the transmission $\left(E_{\text {tran }}\right)$ of an $S$-bit data by the sensors are used:

$$
\begin{aligned}
& E_{\text {tran }}(S, d) \\
& =\left\{\begin{array}{c}
S E_{\text {elec }}+S \epsilon_{f s} d^{2} \quad \text { if } d<d_{0} \\
S E_{\text {elec }}+S \epsilon_{m p} d^{4} \quad \text { if } d \geq d_{0}
\end{array}\right.
\end{aligned}
$$

where $E_{\text {elec }}$ indicates the energy dissipated to run electronic devise circuitry. The energy consumption in free-space and multipath fading model are given by $\epsilon_{f s}$ and $\epsilon_{m p}$ respectively. $d$ denotes distance and $d_{0}$ is the threshold value. The receiving energy, $E_{R x}(S)$, for an $S$-bit data for a node is provided as follows:

$$
E_{R x}(S)=S E_{\text {elec }}
$$

To calculate the residual energy of a node $n_{i}$, the following equation is used: 


$$
E_{r e s_{i}}=E_{\text {tot }_{i}}-E_{\text {tran }_{i}}
$$

where $E_{\text {res }}$ is the residual energy, $E_{\text {tot }_{i}}$ is the total initial energy and $E_{\operatorname{tran}_{i}}$ is the transmission energy.

\subsection{Fitness Function}

A function for the evaluation of the routes, path assessing index, is provided here considering the current energy of the nodes, and the path of routing. If remaining energy is not assessed as in the conventional ACO algorithm, which causes early demise of some nodes and ultimately affects the whole network lifetime. The routing path is related to each particular ant after all the ants get to the destination node. The fitness value for the path can be computed as follows:

$$
f_{(f i t n e s s)_{m}^{k}}=\frac{E_{\text {res }}}{L_{m}^{k}}
$$

where the residual energy level of a sensor node $n_{i}$ is $E_{\text {res }} . L_{m}^{k}$ is the length of the route for $m^{\text {th }}$ ant and $k^{\text {th }}$ iteration. The pheromone is updated on the optimum path, having the highest fitness value.

\subsection{Planning of Route Phases}

Step 1. The route's arrangement phase. At first an initialization signaling is broadcasted by the sink node. Each node acquires its own neighboring node, then updates and adds it to its own routing table. The adjacent node link pheromone value is set to 1 . The current node's ant packet is generated by each node that contains the number of nodes and the routing table. $N_{\max }$ is set to the maximum number of iterations, and the initial number of iterations is set to 1 .

Step 2. The route's organization phase. The next node will be selected by the ant that locates in node $i$ according to (1)-(2). Upon receiving the ant package, a node forwards ant package in accordance with the probability $P_{i j}^{m}$.

Step 3. The route's optimization phase. Once all ants get to the destination node, i.e. the sink node, the fitness function is used for optimal route selection where the fitness value is computed for the route according to (9) and the route with highest fitness value is chosen for optimal route for data transmission. The concentration of pheromones on this path is then updated according to (3)-(5).

In this route's optimization phase, once the ant package is received by the sink node, it will count how many ants packets each node sends. Let's assume that the total node number is $n$ and the number of ants package sent by, for instance, node $i$ is $X_{i}(i=1, \ldots \ldots, n)$.

Then the total sum of the network ant package can be expressed by means of:

$$
T=\sum_{i=1}^{n} X_{i}
$$

Back ant package is generated by each node. When back ant package is received by the nodes, the back ant package's adjacency linkage information is updated by (3)-(5). The node is selected as the next hop which resides in the back ant package. Following the information in the back ant packets, it is determined whether to send a new packet of ants at that same instance. When new ant package need to be sent, the route is established.

\subsection{Proposed Improvement}

Forming clusters associated with the group of nodes is deliberated on achieving scalability and robustness. For this reason, the routing protocol presented in [20] can be integrated with a clustering routing technique, such as LEACH (Low-energy adaptive clustering hierarchy) protocol [22]. It improves the selection strategy of optimal cluster head $(\mathrm{CH})$, which is based on probability, node residual energy, and the distance of a node from the base station (BS). For selecting the cluster head node $j$, if it is assumed that node $i$ is the current cluster head and next node is $j$, an ant $m$ will use the probability calculation given by:

$$
\operatorname{Prob}_{i}(\mathrm{t})=\frac{\operatorname{dist}_{i} * \alpha+\left[P_{i j}^{m}(t)\right] * \beta}{\sum_{1}^{N_{i}} \operatorname{dist}_{i} * \alpha+\left[P_{i j}^{m}(t)\right] * \beta}
$$

Where $\operatorname{Prob}_{i}$ provides the probability of each node to be selected as a $\mathrm{CH}$ (cluster head), dist ${ }_{i}$ is the distance of node, $\alpha$ and $\beta$ are two control parameters and $N_{i}$ denotes the set of cluster nodes. $P_{i j}^{m}$ is found from (1). Figure 1 shows the flow diagram of this suggested enhancement.

Using the proposed ACO based routing algorithm and the proposed improvement given above the initial optimal $\mathrm{CH}$ (cluster head) is selected. Then the final optimal $\mathrm{CH}$ is elected using the maximum fitness function value provided in (9). The optimal initial cluster head sends data to the optimal final cluster head. The optimal final cluster head transmitted data to the sink node.
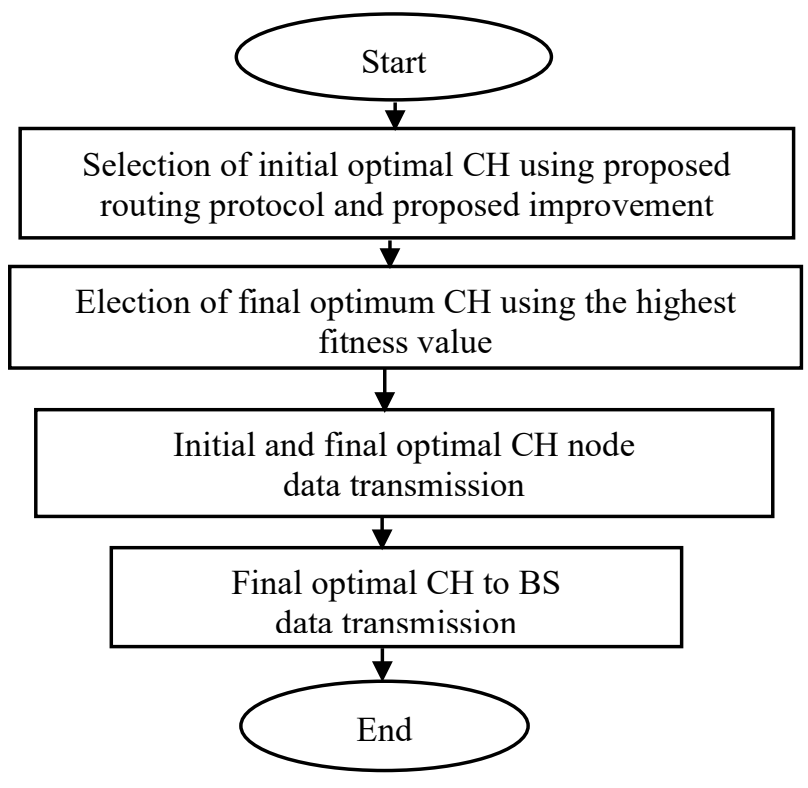

Figure 1: The flow diagram of the proposed improvement

Figure 2 shows the flow diagram of the proposed routing protocol operation.

\section{Result and Discussion}

The simulation is performed utilizing MATLAB where the proposed routing protocol is evaluated according to some important parameters, i.e., the consumed energy, the lifetimes of nodes, best cost, end-to-end delay, throughput and packet delivery 
rate. The method represented here is evaluated against the conventional ACO, a current routing protocol for IoT, e.g., RPL (routing protocol for low power lossy network) [23] and an existing ant colony based algorithm, EICAntS algorithm [12] as benchmark protocols.

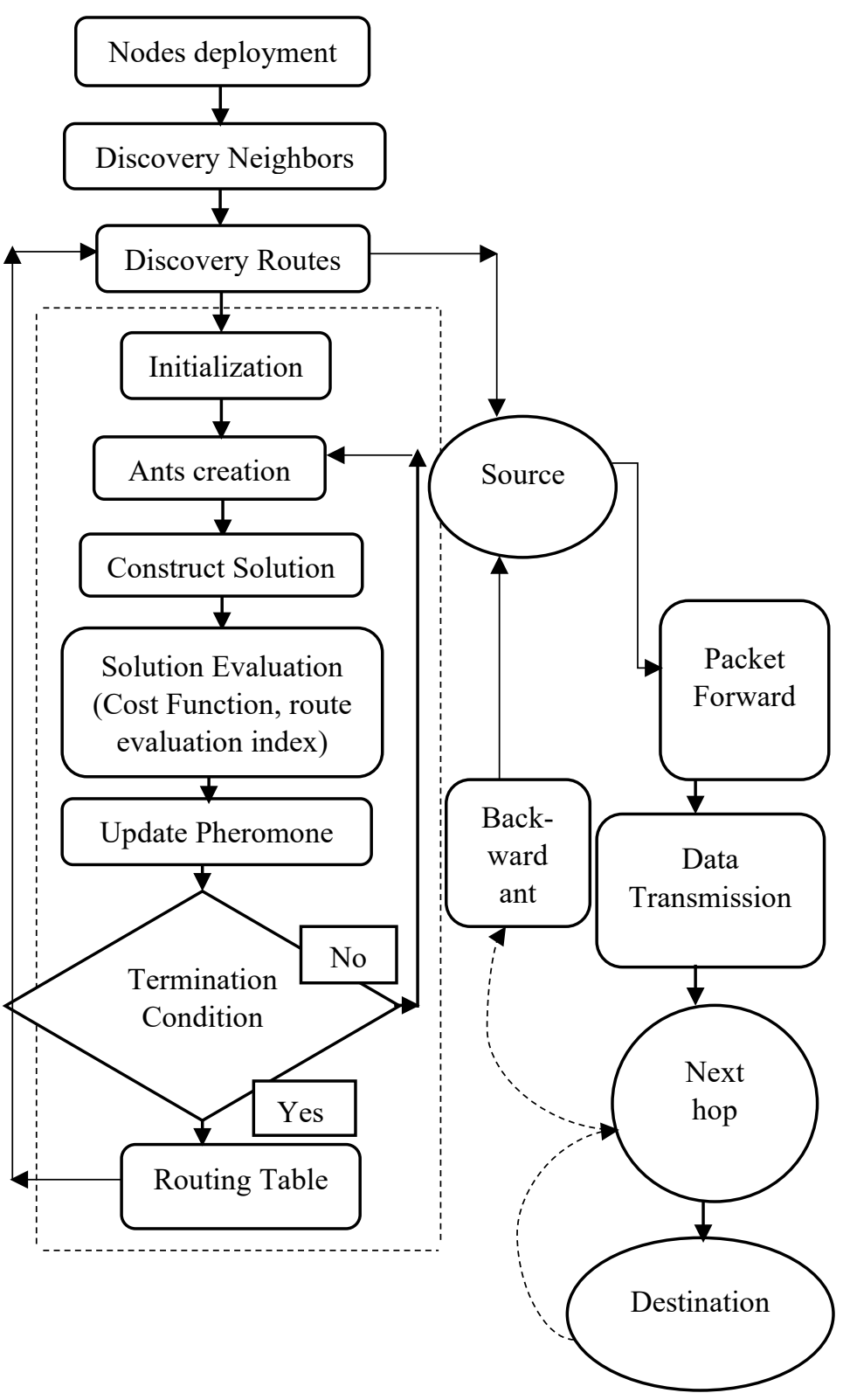

Figure 2: Overview Block Diagram demonstrating the Proposed ACO based Routing Algorithm Operation

The simulation parameters are based on the repeated tests and on the basis of simulation analysis of various parameters and set as: $\alpha=1, \beta=1, \gamma=1, \rho=0.05$. More parameters are provided in the Table 1 .

The proposed system is supporting a mechanism permitting the selection of the best cost route for information communication from the source node to the destination, the base station. The network's parameters are dynamically adjusted to the network evolution, the growing number of nodes, the changed environment issues due to mobility, and outstanding node energy.
Table 1: Simulation Parameters

\begin{tabular}{|l|l|}
\hline \multicolumn{1}{|c|}{ Parameters } & \multicolumn{1}{c|}{ Values } \\
\hline Simulation Region & $100 \mathrm{~m} \times 100 \mathrm{~m}$ \\
\hline Number of Nodes & $40,50,60,70,80,90,100$ \\
\hline$T$ & 100 \\
\hline No. of ants & 40 \\
\hline Initial energy per node & 0.5 joule \\
\hline Node speed & $2 \mathrm{~m} / \mathrm{s} \sim 5 \mathrm{~m} / \mathrm{s}$ \\
\hline Message bits transmitted & $4000 \mathrm{bits}$ \\
\hline Transmission distance & $50 \mathrm{~m}$ \\
\hline
\end{tabular}

In Figure 3, for the proposed ACO based routing protocol and for the traditional ACO based protocol, the comparison of the node energy consumption per transmission is shown. The considerable improvement is shown for the proposed method. For majority of the nodes, contrasted with the conventional protocol, the proposed method has smaller energy consumption, almost 50\% less. The proposed calculation has presented noticeable refinement for the total energy consumed of each node for individual discovery for the routes, contrasted with the benchmark protocol.

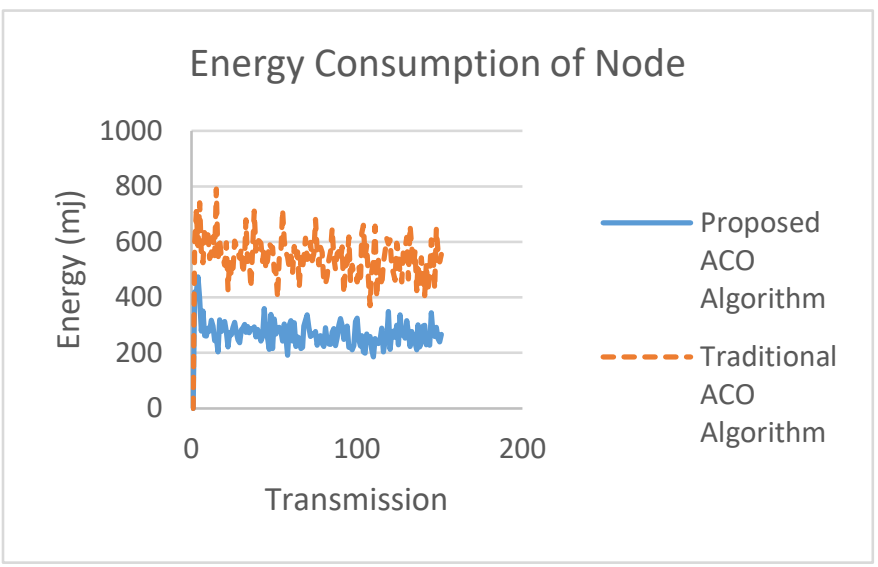

Figure 3: Comparison of node energy consumption per transmission

\section{Node Energy Consumption}

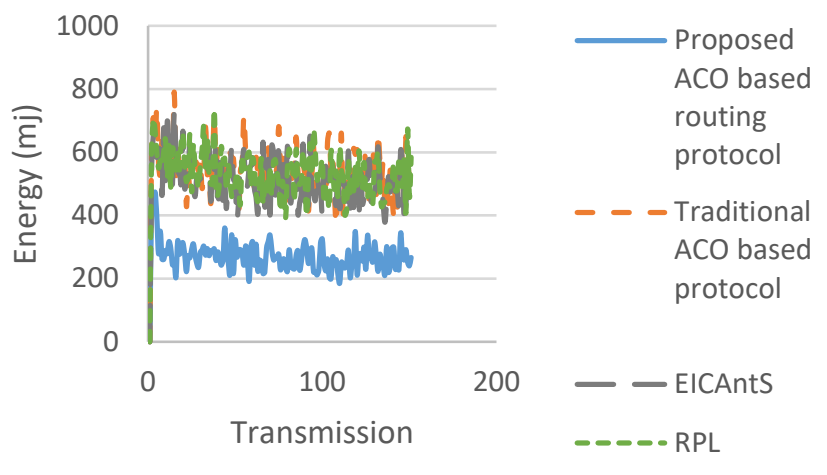

Figure 4: Comparison of node energy consumption per transmission

From Figure 4, we can see the node energy consumption per transmission based on improved method proposed here and other benchmark protocols, based on original ACO, RPL and EICAntS protocol. It is demonstrated from the evaluation results that 
compared with the other two algorithms, the proposed ACO algorithm has attained a refinement, a much smaller energy consumption, almost $50 \%$ less.

The average energy consumption for the increasing quantity of nodes is represented in Figure 5 and Table 2. Figure 5 shows that the system using the proposed ACO based routing protocol as a resolution has minimized the average energy consumption, almost $50 \%$ less, by comparing with the original ant colony-based system, RPL and EICAntS technique. Since the proposed solution allows the determination of the optimal path for packet transmissions, and the retransmissions are avoided as well. Also, this presented method optimizes path selection strategy and minimizes updating phases. The scalability is maintained by the proposed system that has attained lower energy utilization contrasted with the benchmark protocols even when the number of nodes increases in the network.

Table 2: Average Energy Consumption (mj) with the Number of Nodes for 150 transmissions

\begin{tabular}{|l|l|l|l|l|}
\hline $\begin{array}{l}\text { No. of } \\
\text { Nodes }\end{array}$ & $\begin{array}{c}\text { Proposed } \\
\text { ACO based } \\
\text { routing } \\
\text { protocol }\end{array}$ & $\begin{array}{c}\text { Traditional } \\
\text { ACO based } \\
\text { protocol }\end{array}$ & EICAntS & RPL \\
\hline 40 & 270.49 & 545.89 & 516.65 & 532.65 \\
\hline 50 & 281.67 & 570.07 & 566.42 & 574.78 \\
\hline 60 & 303.76 & 620.61 & 606.97 & 632.29 \\
\hline 70 & 330.97 & 650.51 & 660.42 & 679.46 \\
\hline 80 & 350.65 & 686.47 & 695.87 & 692.01 \\
\hline 90 & 372.42 & 739.92 & 744.55 & 735.55 \\
\hline 100 & 401.21 & 791.33 & 799.01 & 768.49 \\
\hline
\end{tabular}

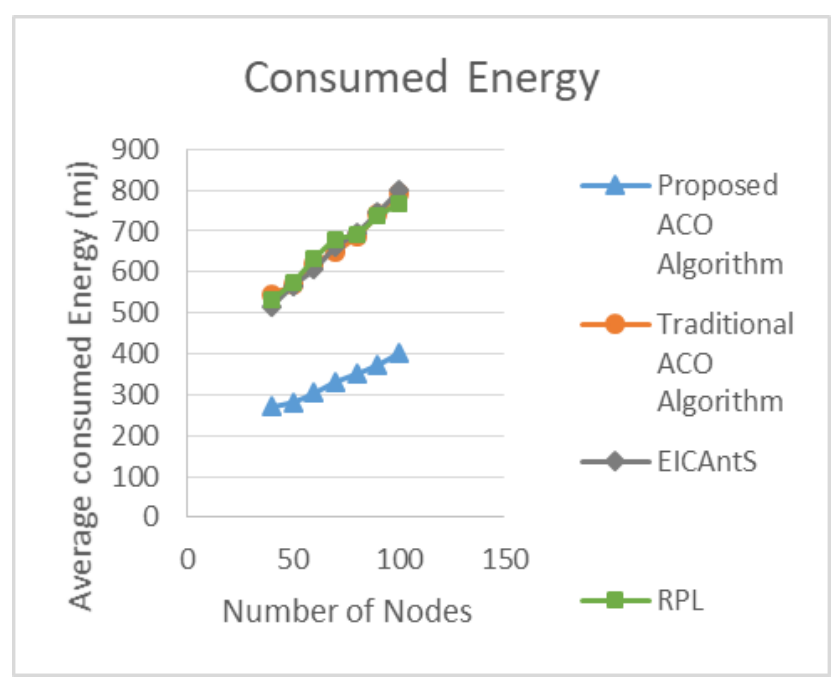

Figure 5: Comparison of the average energy consumption
The effective use of energy lead to increase of network lifetime. The percentage of survival nodes based on improved protocol proposed here and the traditional ACO based protocol are shown in Figure 6 and 7. The issues of ineffectiveness and uncertainty of the routes are alleviated as the protocol proposed here takes into account the energetic parameters. The energy utilization needs to be balanced and the present node energy needs to be deliberated; otherwise, the node energy is depleted and some nodes expire. The network lifetime is affected by this. The proposed approach deliberates the existing energy of the nodes besides the route path. Employing the suggested enhancement according to (11), the longevity, i.e. the nodes' lifetime and lifetime of the network, is increased by the offered ACO based technique.

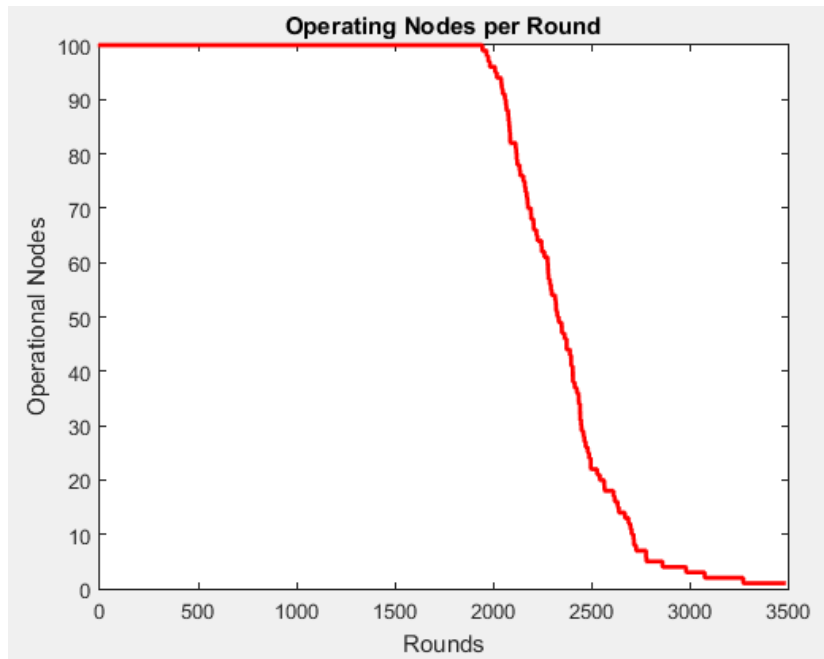

Figure 6: The percentage of survival nodes for the proposed method

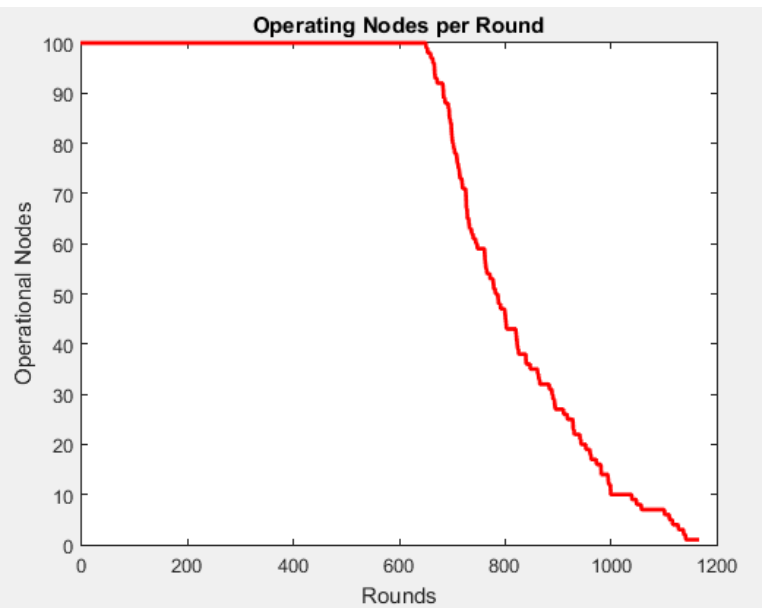

Figure 7: The percentage of survival nodes for the traditional ACO based protocol

We can see from the Table 3 and in the Figure 8 that the average end-to-end delay increases with an increase in the number of nodes. Route discovery and path selection stages affect this parameter, which are instated all the more regularly with an imperative number of nodes and with the topology evolution because of mobility of the nodes. Using the proposed algorithm the route discovery and selection stages are improved and the repetition problem is minimized when many packets should be resent to the destination in case the route might not be the best 
always. The proposed system achieved better results with regard to average end to end delay, almost $40 \%$ reduced end to end delay, compared to the other benchmark techniques.

Table 3: Average End-to-End Delay (ms) with the Number of Nodes using Fitness Function

\begin{tabular}{|c|c|c|c|c|}
\hline $\begin{array}{c}\text { No. of } \\
\text { Nodes }\end{array}$ & $\begin{array}{c}\text { Proposed } \\
\text { ACO based } \\
\text { routing } \\
\text { protocol }\end{array}$ & $\begin{array}{c}\text { Traditional } \\
\text { ACO based } \\
\text { protocol }\end{array}$ & EICAntS & RPL \\
\hline 40 & 159.78 & 367.41 & 364.42 & 370.67 \\
\hline 50 & 203.59 & 397.57 & 394.64 & 382.81 \\
\hline 60 & 250.81 & 413.22 & 412.96 & 410.75 \\
\hline 70 & 263.37 & 427.28 & 414.09 & 425.13 \\
\hline 80 & 299.25 & 464.68 & 460.82 & 455.42 \\
\hline 90 & 312.43 & 478.52 & 465.4 & 466.35 \\
\hline 100 & 327.46 & 485.86 & 479.72 & 488.96 \\
\hline
\end{tabular}

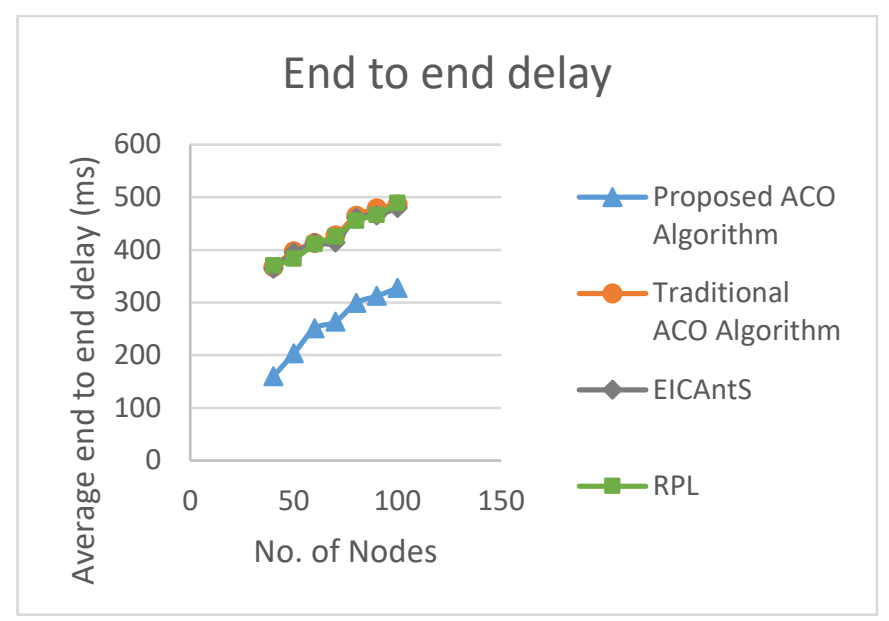

Figure 8: Evolution of average end to end delay with the number of nodes using fitness function

Throughput results are presented in Table 4 and depicted in Figure 9. The quantity of digital data, over a physical or logical connection per time unit, is the measure of the throughput in sensor networks. It is measured in bits/s or bps (bits per second), occasionally in per-second data packets or in per time-slot data packets. It can be defined mathematically, as the total number of packets delivered over the total simulation time:

Throughput $=\mathrm{N} /$ Total simulation time

where $\mathrm{N}$ is the number of bits received successfully by all destinations.
We can clearly see from the contrast that the results obtained are better than the results recorded by the other network.

Table 4: Throughput with the Number of Nodes over the Total Simulation Time

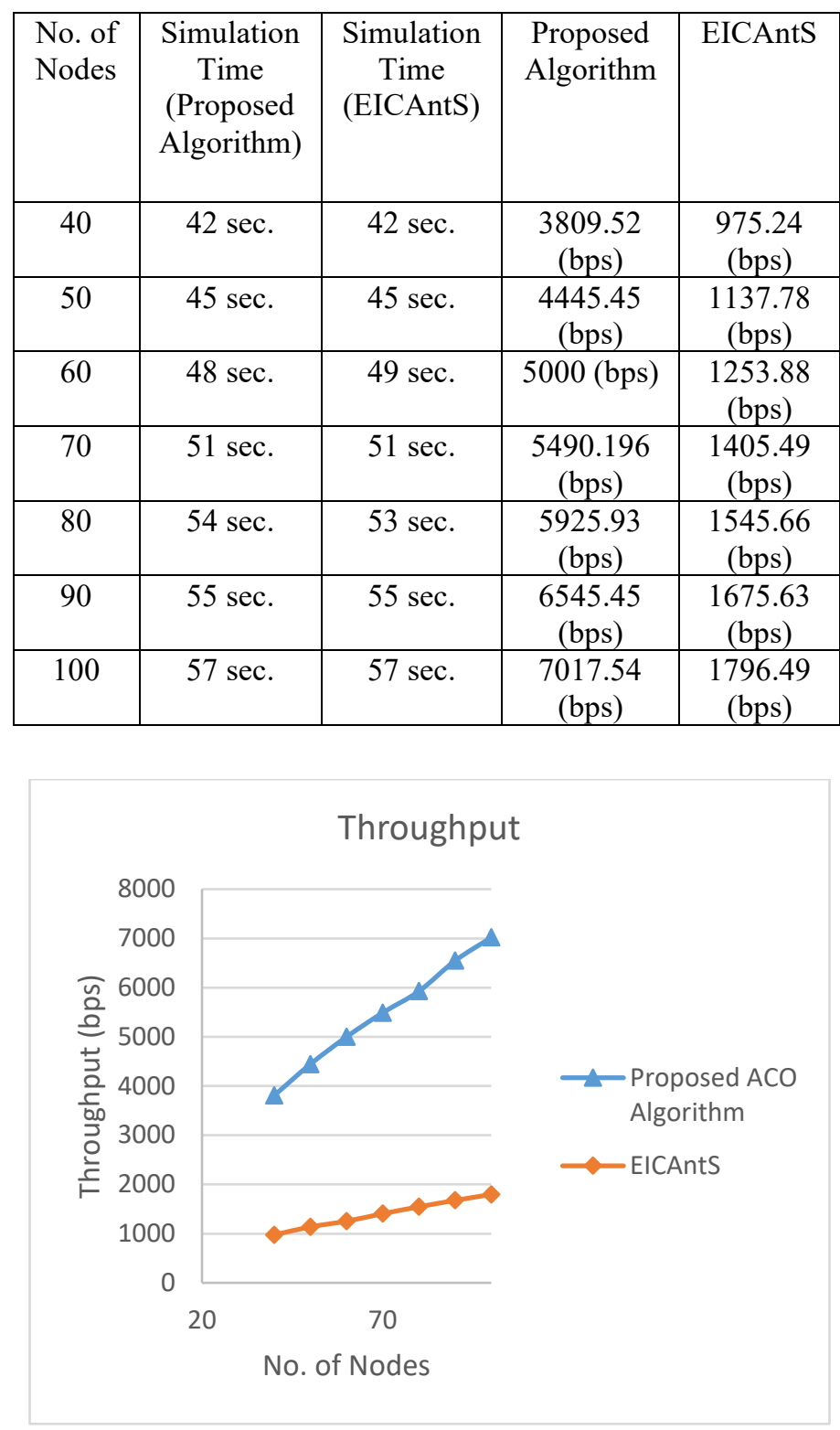

Figure 9: Throughput for the Proposed ACO and EICAntS algorithm

The calculation of the proposed algorithm in accordance with the delivery rates of the packets is provided in the Figure 10. The good results found with the proposed approach, even with an imperative number of nodes because the system has the capability that let many packets to send to get to the destination node in short time.

We can see from the comparison of the best cost as presented in Figure 11 that the network using proposed protocol has minimized initialization delay compared to the benchmark protocols. The network system achieved faster convergence for the initialization delay to discover the best cost route, which is about $30 \%$ improvement in convergence. Current node energy, mobility, route path, and cost function including route assessment index are 
considered by the proposed method, making it preferable as a solution for the network system to improve communication quality. Figure 12 shows that utilizing the route evaluation calculation, about $60 \%$ enhancement can be recorded to find the optimum path with faster convergence in initialization delay.

\section{Packet Delivery Ratio for the Proposed Algorithm}

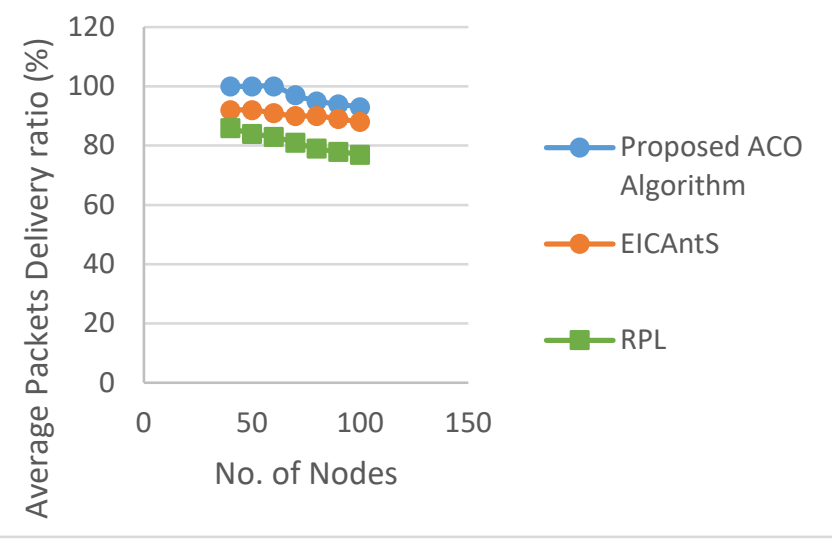

Figure 10: Packet Delivery Rates with the Number of Nodes

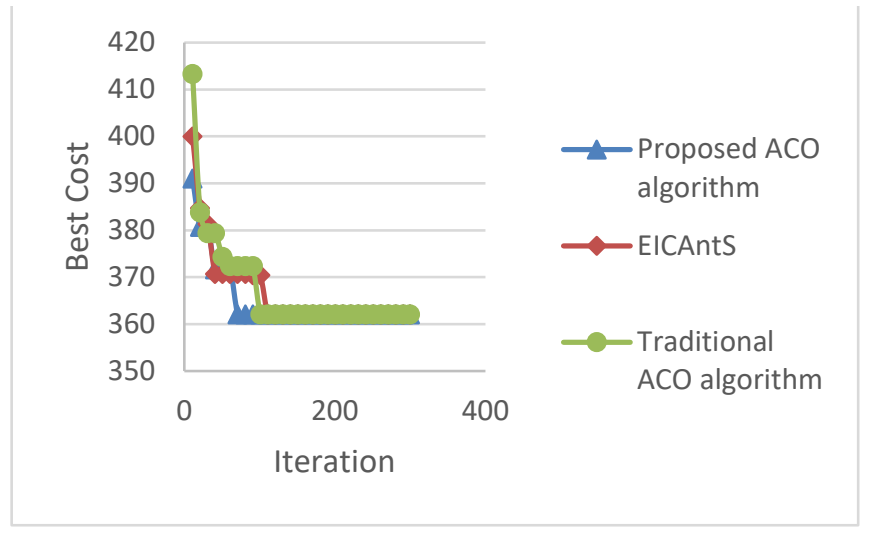

Figure 11: Comparison of the best cost with respect to the number of iterations (not utilizing fitness function)

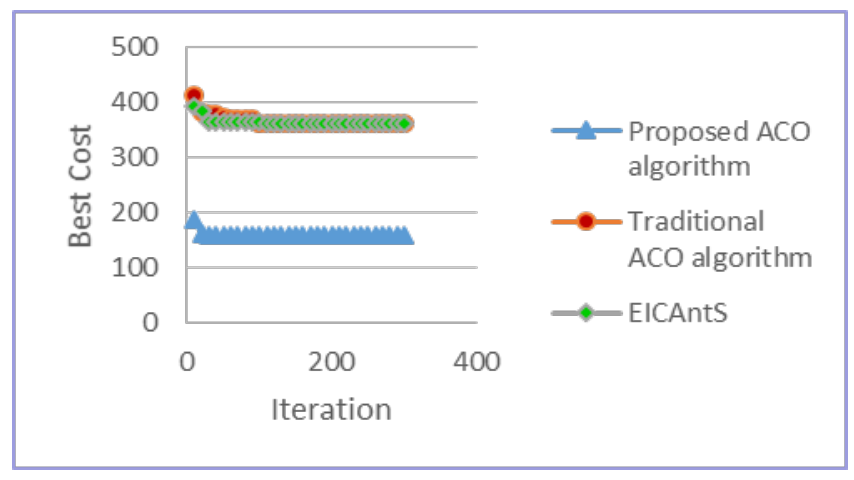

Figure 12: Comparison of the best cost with respect to the number of iterations using fitness function

\section{Conclusions}

The popularization of IoT-connected devices enabling development of IoT applications possess multiple and complex aspects of IoT while designing efficient communication protocols. The proposed system has utilized the beneficial features of an ant colony system to improve the route determination process and communication quality and mechanism in the context of IoT and to make IoT network system more scalable under varying load conditions. The parameters used in the selection of the routes are dynamically attuned to manage the network's dynamic condition. Current node energy, mobility, route path, and cost function including route assessment index are considered by the proposed method to enable efficient information communications within IoT. In the probability formula of ACO algorithm, the energy factor and the average mobility of the nodes are incorporated as well. It is demonstrated from the evaluation results that the proposed ACO based routing algorithm attained faster convergence for the initialization delay, about $30 \%$ improvement to discover the optimum route, and reduced energy consumption, of almost $50 \%$ less consumed energy even with the increasing number of nodes, compared with the benchmark algorithms. The proposed system achieved better results in terms of average end to end delay, almost $40 \%$ reduced end to end delay, compared to the other standard protocols. Also, it is confirmed that the proposed protocol as energy efficient method even for scalable networks, where the performance does not deteriorate abruptly as the number of nodes increases. Rather, it maintained improved network performance comparing with the other algorithms from the benchmark papers.

\section{References}

[1] A. Sharmin, F. Anwar, S.M.A. Motakabber, "Energy-Efficient Scalable Routing Protocol Based on ACO for WSNs," 2019 7th International Conference on Mechatronics Engineering, ICOM 2019, 1-6, 2019, doi:10.1109/ICOM47790.2019.8952053.

[2] Cisco, How the Next Evolution of the Internet Is Changing Everything, White paper, 2011, CISCO White Paper, 2011.

[3] Y.A. Malkani, L. Das Dhomeja, "Secure device association for ad hoc and ubiquitous computing environments," in 2009 International Conference on Emerging Technologies, ICET 2009, 2009, doi:10.1109/ICET.2009.5353132.

[4] I.F. Akyildiz, W. Su, Y. Sankarasubramaniam, E. Cayirci, "Wireless sensor networks: A survey," Computer Networks, 2002, doi:10.1016/S13891286(01)00302-4.

[5] K. Sohraby, D. Minoli, T. Znati, Wireless Sensor Networks: Technology, Protocols, and Applications, 2006, doi:10.1002/047011276X.

[6] G. Anastasi, M. Conti, M. Di Francesco, A. Passarella, "Energy conservation in wireless sensor networks: A survey," Ad Hoc Networks, 2009, doi:10.1016/j.adhoc.2008.06.003.

[7] W. Dargie, C. Poellabauer, Fundamentals of Wireless Sensor Networks: Theory and Practice, 2011, doi:10.1002/9780470666388.

[8] M. Saleem, G.A. Di Caro, M. Farooq, "Swarm intelligence based routing protocol for wireless sensor networks: Survey and future directions," Information Sciences, 2011, doi:10.1016/j.ins.2010.07.005.

[9] T. Gui, C. Ma, F. Wang, D.E. Wilkins, "Survey on swarm intelligence based routing protocols for wireless sensor networks: An extensive study," in Proceedings of the IEEE International Conference on Industrial Technology, 2016, doi:10.1109/ICIT.2016.7475064.

[10] R.A.L. Rabelo, J.V.V. Sobral, H.S. Araujo, R.A.R.S. Baluz, R.H. Filho, “An approach based on fuzzy inference system and ant colony optimization for improving the performance of routing protocols in Wireless Sensor Networks," in 2013 IEEE Congress on Evolutionary Computation, CEC 2013, 2013, doi:10.1109/CEC.2013.6557967.

[11] M. Dorigo, V. Maniezzo, A. Colorni, "Ant system: Optimization by a colony of cooperating agents," IEEE Transactions on Systems, Man, and Cybernetics, Part B: Cybernetics, 1996, doi:10.1109/3477.484436.

[12] S. Hamrioui, P. Lorenz, "Bio inspired routing algorithm and efficient communications within IoT," IEEE Network, 2017, doi:10.1109/MNET.2017.1600282.

[13] K. Machado, D. Rosário, E. Cerqueira, A.A.F. Loureiro, A. Neto, J.N. de 
Souza, "A routing protocol based on energy and link quality for internet of things applications," Sensors (Switzerland), 2013, doi:10.3390/s130201942.

[14] V. Gupta, S.K. Sharma, "Cluster head selection using modified ACO," Advances in Intelligent Systems and Computing, 2015, doi:10.1007/978-81322-2217-0 2.

[15] R. Khoshkangini, S. Zaboli, "Efficient Routing Protocol via Ant Colony Optimization ( ACO ) and Breadth First Search ( BFS )," International Conference on Internet of Things (IThings 2014), (March), 375-381, 2014, doi:10.1109/iThings.2014.69.

[16] M.D. Devi, K. Geetha, K. Saranyadevi, "Content Based Routing Using Information Centric Network for IoT," in Procedia Computer Science, 2017, doi:10.1016/j.procs.2017.09.145.

[17] R. Bao, H. Pan, Q. Dong, L. Yu, L. Shao, "Ant colony-based routing algorithm for wireless sensor networks," Chinese Journal of Sensors and Actuators, 24(11), 1644-1648, 2011, doi:10.3969/j.issn.10041699.2011.11.025.

[18] A. Nayyar, R. Singh, "IEEMARP- a novel energy efficient multipath routing protocol based on ant Colony optimization (ACO) for dynamic sensor networks," Multimedia Tools and Applications, 2019, doi:10.1007/s11042019-7627-z.

[19] Y.L. Wang, M. Song, Y.F. Wei, Y.H. Wang, X.J. Wang, "Improved ant colony-based multi-constrained QoS energy-saving routing and throughput optimization in wireless Ad-hoc networks," Journal of China Universities of Posts and Telecommunications, 2014, doi:10.1016/S1005-8885(14)60267-3.

[20] A. Sharmin, F. Anwar, S.M.A. Motakabber, "A Noble Approach of ACO Algorithm for WSN," Proceedings of the 2018 7th International Conference on Computer and Communication Engineering, ICCCE 2018, 152-156, 2018, doi:10.1109/ICCCE.2018.8539295.

[21] A. Sharmin, F. Anwar, S.M.A. Motakabber, "A novel bio-inspired routing algorithm based on ACO for WSNs," Bulletin of Electrical Engineering and Informatics, 2019, doi:10.11591/eei.v8i2.1492.

[22] W.B. Heinzelman, A.P. Chandrakasan, H. Balakrishnan, "An applicationspecific protocol architecture for wireless microsensor networks," IEEE Transactions on Wireless Communications, 2002, doi:10.1109/TWC.2002.804190.

[23] S.S. Solapure, H.H. Kenchannavar, "Design and analysis of RPL objective functions using variant routing metrics for IoT applications," Wireless Networks, 26(6), 4637-4656, 2020, doi:10.1007/s11276-020-02348-6. 